\title{
Towards Participatory Video 2.0
}

\author{
Delvin Varghese \\ Action Lab, Monash \\ University \\ Melbourne, Australia \\ delvin.varghese@monash.edu
}

\author{
Patrick Olivier \\ Action Lab, Monash \\ University \\ Melbourne, Australia \\ patrick.olivier@monash.edu
}

\author{
Tom Bartindale \\ Action Lab, Monash \\ University \\ Melbourne, Australia \\ tom.bartindale@monash.edu
}

\begin{abstract}
Participatory video (PV) is an established practice for enabling communities to "speak truth to power" and has been widely used by local, national and international Non-Governmental Organizations (NGOs). However, the digital media landscape has changed dramatically since PV became widely accessible with the rise of the camcorder in the 1980s. Current media practices have evolved considerably since, yet PV remains essentially unchanged. We report on an investigation of current PV practices and reflect on these in terms of what the future for PV holds. We conducted interviews with staff at a global humanitarian network who directly and indirectly engage in community story capture; and explore their reflections on the potentials and barriers to PV use. We propose a new vision for PV that draws on current visual media production, consumption and distribution technologies and practices, and propose principles on which PV 2.0, a new generation of Participatory Video can be founded.
\end{abstract}

\section{Author Keywords}

participatory video; International Development; HCI4D; ICTD; participatory film-making; object based media; iDocs;

\section{CCS Concepts}

-Human-centered computing $\rightarrow$ Human computer interaction (HCI);

\section{INTRODUCTION}

There has been increased interest in the use of video to represent marginalized populations. NGOs have been one sector to realize this potential to engage communities in feedback, peer-to-peer learning and advocacy activities. Such activities are often referred to as Participatory Video (PV), or Participatory Film-making. Like most production methods, $\mathrm{PV}$ is a resource intensive process. In many contexts, significant challenges exist in deploying PV, such as the prohibitive infrastructure and skills required to facilitate the process: video capture equipment, editing suites and staff skilled in production processes. However, over the last two decades, the media

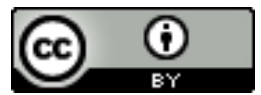

This work is licensed under a Creative Commons Attribution International 4.0 License.

CHI'20, April 25-30, 2020, Honolulu, HI, USA

(C) 2020 Copyright held by the owner/author(s).

ACM ISBN 978-1-4503-6708-0/20/04.

DOI: https: //doi.org/10.1145/3313831.3376694 production and consumption landscape has evolved rapidly and many of the technological and procedural assumptions that underpinned the design of PV practices have changed. This new landscape blurs the line between consumers and producers of content (media), resulting in new challenges for NGOs in producing and utilizing digital media. Content is consumed across time and space on multiple devices, and content is repurposed, reused, reformatted and recycled for many other purposes. This is a complex and ever changing fabric of technologies, practices and processes. Film and video production is no longer the exclusive realm of trained professionals using prohibitively expensive tools, sophisticated technologies and accompanied by insider language. We are living in what has been referred to as the age of the prosumer [34], in which audiences (not just in Western contexts) are sophisticated consumers of content across different platforms in myriad forms. These practices were simply unimaginable at PV's conception in the 60s. For a variety of reasons, PV practice has not evolved to reflect the new challenges and opportunities afforded by increasingly media-literate, technology-literate populations around the world. However, HCI for Development (HCI4D) researchers recognize the potential afforded by the increasing ubiquity of digital technologies in NGO contexts and the increased media literacy of Development communities as as a result.

The mainstream media industry is placing less focus on linear broadcast content, and as content expectations and consumption patterns change, new processes and workflows must be developed to meet the production demands of this content. Object Based Media (OBM) has emerged as a helpful lens through which this new landscape can be understood and designed for. OBM promotes the inherent value of rich meta-data which sits alongside content as 'objects' [29]. Representing media in this way: as reusable elements of self-describing content, reveals opportunities for new consumption models. Examples include second-screen content [28], interactive narratives [21] or interactive documentaries [22, 18] and production workflows (such as distributed or time-lagged production) [28]. We see a unique opportunity to reinvigorate PV as a core practice within this emergent sphere of media.

In light of the opportunities afforded by OBM and the potential of PV in this emergent landscape, we present a new vision for PV use in NGO contexts: PV2.0. To inform this new set of principles for PV, we interviewed both experienced and new practitioners from a large international NGO who directly and indirectly engage in community story capture. With a presence 
in over 120 countries, the NGO is well known for utilizing PV in community engagement with marginalized communities, having worked with several established PV facilitators over the last two decades. Through our inquiry about the perceptions and experiences of current PV practice, our findings indicate i) a lack of engagement within the Development and NGO sector with broader changes in the media landscape for both producers and consumers of content, and ii) opportunities for HCI to leverage emergent practices and understanding from industry to better engage stakeholders in reporting and advocacy of issues concerning marginalized communities. We present this vision as a reflection on the ethical, cultural, economic and institutional challenges currently faced by the organization we worked with, drawing out clear principles that PV2.0 should enshrine as we move towards Object Based production of PV.

\section{VIDEO FOR COMMUNITY ENGAGEMENT}

Global video trends highlight the increasing engagement of populations from Development contexts with video, through social media and digital services such as YouTube. NGOs, cognizant of these changes in consumer behavior, are recognizing the importance of utilizing media in new ways in their organizational processes. Participatory Video (PV), is a specific application area of this synergy between NGOs and visual media, which has historically been used by organizations within such contexts.

\section{Modern Video Practices \& Trends}

The nature of video consumption worldwide has changed dramatically in the last decade. According to GlobalWebIndex, $92 \%$ of internet users now watch videos online each month (roughly 4 billion people in early 2019) [16]. By comparison, 6 billion people around the world have a television set at home [16]. Web 2.0 saw the proliferation of social media and the shift from media being broadcast by "experts' to usergenerated distribution and consumption of content [47] as a common practice. Video has increasingly been the medium of choice for contributors, aided by ubiquitous devices and platforms that promote a video-first approach (a phrase coined by Mark Zuckerberg in 2017) [24].

It comes as no surprise that mobile phones are the most popular device for watching videos worldwide [17]. Indeed in the AsiaPacific region, consumption on mobile devices accounts for $74 \%$ of all video plays, up $64 \%$ in two years. Similarly, in Latin America, mobile video views topped 65\%, an increase of $20 \%$ year-over-year and $38 \%$ over two years [42,33]. It has been forecast that by the end of 2019 , video will make up $80 \%$ of all internet traffic [42]. Noting these trends, we argue that in light of the fundamental shift brought about by this changing media landscape, film-making approaches being applied in NGO field contexts need to be better prepared to address the emergent challenges and opportunities of mobile and media literacy.

Studying the radical implications of mobile video growth on the US cable TV industry, Bentley and Lottridge set out to understand how the industry reflected American mobile viewing practices and set out design considerations [4]. Similarly, we seek to understand the expectations and challenges of PV experienced by NGO staff who take part in community engagement activities towards developing a new vision for PV that better suits the emergent media landscape.

\section{Supporting NGO Practices Within HCI4D}

There is a rich history of using video to support NGO Practices within HCI4D. Kumar et al. collaborated with a rural Indian NGO to deliver locally created instructional videos about maternal healthcare [27]. They noted that participants had to learn many skills beyond video capture and production, including the ability to handle sensitive issues, and noted the significant time it took to gain such skills [27]. Similarly, the educational level of participants has been found to be a key factor in participants' ability to use interfaces to access video content [9]

Indeed the seminal work of Frohlich et al. [15, 14] explored the use of mobile phones by marginalized populations to create and share audio-visual content, although we note that the camera phones available in 2009, when the author conducted this study were limited in their audio-visual capabilities by modern standards. This approach of using consumer technologies is in contrast to that of Kumar et al., who used professional video capture equipment. Frohlich noted the propensity of participants to embrace mobile and digital storytelling approaches but added that this required a very simplified form of story and infrastructure [15]. These two examples provide contrasting views of facilitating video-work in NGO field contexts. As such, the complexity of the video process and the infrastructure available can dictate the amount of on-hand support required by participants and how they make sense of the data.

There are myriad stakeholders within Development including NGOs, local volunteers, national and local government actors, donors, and community members (beneficiaries). Filmmaking as an activity has gained popularity as it helps address one of the key concerns within HCI4D research: not all stakeholders may be represented equally when reporting on interventions that affect them. This is not to say that the nature of participation PV affords community members is not contested. These are all part of broader debates about participant representation, power, and vulnerability [48]. Indeed, previous researchers have advocated for an "ethical need to acknowledge the messy reality of the PV context" [43], and we agree that any new vision of PV needs to acknowledge these issues at a fundamental level and be designed to expose, support and value these considerations.

\section{PV: A Rising Star in HCl4D}

Low and Snowden's Fogo process in 1967, put film-making equipment directly in the hands of children [35, 31], capturing the challenges of living on Fogo Island, a Canadian island in the province of Newfoundland. Since then, a number of organizations (NGOs in particular), have seen the potential in using PV to enable the communities they work with to tell their stories. Participatory Filmmaking is another term that is frequently used to refer to the activities conducted under the PV umbrella. Reflecting on these two terms, PV might arguably be seen as a misnomer: the video shouldn't be the focus, it is the process of storytelling and the act of film-making that is 
central to the ethos of what constitutes PV. Other researchers have discussed the challenges associated with using PV as a medium to engage participants. In Manuel et al.'s work [31], PV was used to facilitate the capture of citizen stories towards a local neighborhood planning process. Participants used PV to shed light on previously hidden narratives, which highlighted elements that usually get lost behind when participatory processes rush towards a defined output. An important debate within PV has been about how the process is conducted, and where the 'data' lies. While superficially, the output of a PV process is the video, there has been a chorus of voices noting that the process of conducting PV can be just as rich and yield important findings about the community's thinking and perception of their problems and solutions offered by the NGO. PV therefore can support empowerment and agency building, within NGO field contexts through the process itself. However, it is no panacea, sometimes existing systemic forces of oppression are left masked within PV outputs, even if they are laid bare by the community in the dialogues and discussions in the PV process [39].

In response to these and other challenges, Bartindale et al. [3] discussed the use of PV approaches with the Red Cross movement. They noted that by leveraging the advancements in mobile technologies, barriers to entry for video work could be significantly reduced. Building on their work, we argue that the NGO field context offers a number of complexities and real world challenges that are key to a better theoretical understanding of the potential and challenges for video work in HCI. In the next section, we describe our engagement with a global NGO about their staff's experiences with PV. We reflect on their views in light of contemporary video practices, critically assessing the benefits and drawbacks of current PV approaches.

\section{CONTEXT}

Over the last three years we have conducted video work with various Development organizations including IFRC who are increasingly using PV based approaches in their work with communities across the world. They are based in over 100 countries around the world, and are key actors in both shortterm (emergency) and longer-term (development) interventions. The contexts in which they work present a number of challenges to the wider adoption and utilization of PV throughout the network. This motivated us to speak further with their staff who are based across the world in various community feedback and community engagement roles. In this research, we look particularly at how this NGO goes about doing community engagement work, particularly within the sector of Monitoring and Evaluation. This has been informed by previous research within the CHI community, looking at feedback elicitation from communities in general, and project monitoring within International Development specifically. There are many motivations for organizations to engage communities in project evaluation and feedback gathering. It is vital for these NGOs to understand the impact of their programs on communities, especially when they are working with them longitudinally, particularly as feedback given directly by communities is increasingly seen as more credible and real for donors and other stakeholders.
Monitoring and Evaluation (M\&E) processes help organizations use "relevant information from past and ongoing activities that can be used as the basis for programmatic finetuning, reorientation and future planning" [49]. Without such processes, stakeholders do not have an effective heuristic on which to base their assessment of the success or failure of ongoing activities. In the words of one experienced M\&E practitioner working for $I F R C$, video-based evaluation methods are required if, "from the eyes of the community, if you want to see what's working and what's not working". It is an oft-repeated sentiment, that rich media such as audio-visual methods can be used for a better understanding of community relations and values in terms of Development assistance. The premise of this paper is to assess this statement in the light of the contemporary media landscape, and what new avenues can be explored by NGOs when working with communities.

\section{METHODOLOGY}

We conducted interviews with experienced practitioners with a view to understanding their perceptions and experiences of the use of PV in humanitarian work. To ensure a robust discussion about PV and its nuances, we concentrated on those with previous experience of PV methodologies. While the larger goal of our study was to re-imagine PV in the light of new and emerging media practices (such as Object Based Media), our concern was to maintain a focus on the essential qualities of PV as a tool for community expression - as originally conceived. We were mindful in aligning our questions and subsequent discussion onto current and past experiences of PV, rather than inviting specific reflection on PV in relation on wider contemporary consumer use of video (beyond the realm of current PV practice itself). Participants represented personnel who had either used PV in the field (either through commissioning it, or being involved in the process), were familiar with it's use within the organization (e.g. using the resulting media), or had specifically expressed an interest in using it within their regional portfolio. The wide range of career backgrounds (many had worked for other NGOs previously) and current roles within the organization brought diverse perspectives on the potential and challenges of using PV. Participants consisted of one water, sanitation and hygiene (WASH) Officer [P1], seven M\&E staff [P2,P4$\mathrm{P} 7, \mathrm{P} 9, \mathrm{P} 10]$, three IT \& systems staff [P3,P13,P14], three community engagement officers [P8,P11,P13], one logistical staff member [P15] and one branch manager of a regional NGO chapter [P16]. Five were based in the Asia-Pacific region [P1$\mathrm{P} 3, \mathrm{P} 5, \mathrm{P} 6]$, four in the Southern Africa region [P13-16], three were headquarters-based (Geneva, Switzerland) [P8,P11,P12], two in the Middle-East North Africa region (MENA) [P7,P9] and one staff member each were based in Eastern Europe [P4] and South America [P10].

Semi-structured interviews were conducted and recorded via Zoom $^{1}$. Our collaborator, a senior M\&E staff member based at IFRC headquarters brokered introductions with the participants through the global network. Participants were introduced to the project via email, where they agreed to anonymously sharing their views on PV, its potentials and challenges with

\footnotetext{
${ }^{1}$ https://zoom.us
} 
the research team. Over a three month period, participants were interviewed on their previous PV experience, the types of approaches they had used, the challenges they had faced using PV in their role and their current and historical motivations for using PV. Of the 16 participants, 10 participants were interviewed in pairs. Each interview was transcribed verbatim, and the set was analyzed with an inductive coding approach [46]. A code-book was generated from the first three transcripts that were analyzed, which was refined by using code categories to thematically organize similar codes. This was then used to code the entire data-set, refining the code categories throughout the process.

\section{FINDINGS}

Our analysis yielded six themes relating to: (i) PV relationship to traditional data collection methods (and how its value as data was unrealized); (ii) dispute as to the credibility and practicality of $\mathrm{PV}$ in its current incarnation; (iii) the different ways that PV can mediate interactions between stakeholders; (iv) how to innovate within organizational and financial constraints; (v) motivations for using PV; and (vi) strategies for innovation within the constraints of the organization.

\section{The Unrealized Potential of PV as Data}

$\mathrm{PV}$, as both a medium and a process, was consistently referred to as form of data collection that could represent the complexities of rich field contexts in ways that traditional methods qualitative and quantitative methods could not. Participants repeatedly referenced the agency, creativity and sense of ownership that it affords the communities that they work with. PV was described as coming from "the people who have been affected... so its not [just] stories" [P13].

As well as the "more human touch" [P4] of the medium, it is seen as having an immutable quality, that when "circulated more than a hundred times, you still have the [same] firsthand information" [P13]. Our participants were consistent in their beliefs that "video should not be seen as the only way of doing it" [P5]. In fact they expressed a subtle awareness that PV was a form of data (and data collection) that is not enmeshed with their current organizational practices, and that as such, it offered an alternative to traditional qualitative and quantitative data collection (such as interviews and surveys). Participants noted that PV could be "pretty fun, especially for youth and that people nowadays feel quite excited to use" [P8], with one senior practitioner describing how he "could see that the people can speak freely, they can tell their concerns, give their input, they can complain" [P1]. However, noting that $\mathrm{PV}$ was analogous to the open-ended survey question, which were increasingly less common due to it being "very hard to systematize and to analyze this kind of data" [P10], there was reticence at being able to use this video as 'usable' data.

That is not to say that everything shared openly by communities are uncritically accepted by project staff. We did not find an NGO equivalent of the common aphorism "the customer is always right".

Indeed, some saw the issues a community might raise during PV as something to be managed: "we have to set these kind of boundaries, that 'if you make this video please just focus on the scope of the project alone"' [P1]. But the frankness of responses and critical engagement elicited from communities was identified as something of value, and that they should be sensitive to: "so they did not always do videos on disaster risk reduction, they felt like doing videos on poverty and... Because they felt 'my main issue is I'm poor, it's not disaster risk reduction, I don't even understand what that is anyway!" [P8]. Thus PV allows beneficiaries to talk about their life circumstances using video as an opportunity for advocacy and facilitating more aid (or continued intervention) for themselves. There is a wide awareness on the part of the evaluation staff as to the potential benefits of using PV in this way: "even if not part of our project... it is a good thing because we then have evidence we can refer to other organizations or stakeholders" [P1]. However, despite recognizing PV as having unique qualities over alternative methods, our participant responses were tempered by the need to consider its use in the context of the traditional repertoire of data collection. Using videos is valuable to engage participants who would otherwise find the concepts challenging [52]. Indeed, discussions of the value of PV was entangled with a wariness to champion any data collection method over any other. This entanglement is reflected in accounts of practice, with many participants describing how first-hand video accounts are used as a light touch complement to their everyday methods of data collection; with snippets of PV outcomes being selected to add authenticity and credibility to standard reports: "we include some of the things that people say in the PV evaluation, to make more strong the data that you are presenting. You are putting voice to the data" [P10]. The underlying sentiment is that multimedia outputs such as video are useful for enriching other forms of 'real' data that were collected. Moreover, many of our participants expressed concern that the videos themselves actually constituted an under-utilized source of data.

\section{Bridging Qualitative and Quantitative data}

This under-utilization was often characterized in relation to their ability (or lack of) to systematically analyze the videos: "we're not very well-versed in the methodology of how to analyze it... with mobile data, questionnaires, these are things that we have used, tried and tested" [P6]. Though traditional data collection approaches might have certain weaknesses, the staff's familiarity with them meant that they were more comfortable using them.

On different occasions participants equated the output of a PV process with both qualitative and quantitative data and made reference to ongoing debates about the validity of analysis of such: "There's a lot of [confusion] in past qualitative data collection, where people converted it to quantitative so the analysis is wrong. Unless there is a really good statistician who can analyze video data" [P6]. Concerns about methodological rigor raised issues around a lack of appropriate training, recognizing that existing practitioners "don't know how to use NVivo, ATLAS, and then yet they analyze like 'Ten percent of people from this room say this, they like this very much' it's completely wrong, they can't say that!" [P6]; and the resource constraints that ultimately determine what can be done in monitoring and evaluation activities in the field: "do we have the capacity to do this analysis of all the videos 
that come through?" [P12]. There is a concern that over-use of PV may lead to a volume of qualitative data for which they lacked the capacity to analyze and report on effectively.

The concerns around PV are also focused on whether relying on a qualitative approach may yield more biased data. One respondent worried about the effects of Participant Response Bias [11] if PV were employed for their projects: "this is my assumption, but they can get excited and give socially desirable [answers] or response bias" [P5]. Similarly, concern on bias was centered around the perceived credibility of qualitative approaches, unless they were facilitated by an external organization: in essence auditing the project: "And we didn't want to be the ones really driving this on our own because we felt that it would totally bias what they would report on." [P8].

This highlights a cultural clash within an organization which is moving towards "becoming a data-driven organization", as they progress to harness more quantitative data in their processes. The use of qualitative methods might create more work for practitioners, as "stakeholders will still demand quantitative information". This means that practitioners who try to use non-quantitative data will end up creating more work for themselves, in having to 'convert' the data back to the quantitative data that would be appeciated by the rest of the organization. As such, the organization is keenly aware that the community representation on PV outputs are "just snapshots and they're not necessarily representative of the whole community...its just one perspective" [P8]. There are some open questions remaining then about how the strengths of PV fit into the organizational narrative and challenges about how to use data that it generates. This is one of the key reasons why practitioners are reluctant to endorse PV above other data collection approaches, as "ultimately quantitative data is what appears in our reports...that's what donors want to see, that's what our office wants to see" [P5]. These are not deficiencies within PV methodologies but rather that PV practice is fundamentally at odds with the organizational culture of data collection and analysis.

\section{Participation in Practice}

Different levels of concern about the role of PV facilitators are reflected in our participants views on the credibility of claims to participation. Some viewed PV as just video-work that is done as a group activity, in which the contribution resides in the ability to enable "a group of people [to] share their ideas, their knowledge, their experiences, their lessons learned". Yet one practitioner, who had previous experience facilitating participatory evaluation excercises, reflected on how editing communities' videos, and helping communities to make their video, calls into question the 'participatory' nature of PV: "you'll also need to encourage them a little bit to finish their videos and by doing that you actually influence their process. So I don't know if it can be a hundred percent participatory" [P8]. Another expanded on how an issue in their video practice (not PV) was that the editing and post-production is done (geographically) away from the communities who are actually represented: "the communities are not involved in that process of organizing the information and the ideas that they are presenting" [P10]. For this participant, "that's the most powerful part of the PV evaluations that they do and they produce their videos and they select what they say and for me that is very, very important" [P10].

Aspects of how participation is managed and controlled, and the role these play in mitigating the goal of PV to 'speak truth to power' [32], were a concern for a number of participants: "you're trying to empower them to do their own thing...then afterwards you come and say 'oh no, not that story actually..." [P8]. Participants reported that organizations are understandably very careful in ensuring that communities don't say something incendiary about other stakeholders, particularly local and national government. Many expressed a sense of 'complicity' in curating the presentation of communities in a particular light to other stakeholders: "that's what we do on a daily basis when we take videos of them and then we go to our government and say 'give them money because they're poor' or whatever" [P8]. As such, practitioners were very aware of the tension of perception in their practice, and the role they play in representing a community to other stakeholders.

\section{Social, Cultural and Political Realities}

Tensions surrounding management and control of the process and data extend to the fact that the community "does not control who is actually going to watch that video" and have no control over who the organization promotes or shows the videos to. In one case, a MENA-based practitioner recounted an interaction with a community member: "we told him 'this video is going to be aired in Canada and it will not be shown in [country]' and he said 'OK fine, as long as it does not show in [country] it's fine"' [P3]. This was an experience encountered by our participants who had worked in in regions with political and social conflict. Another practitioner described a context she had worked in: “you wouldn't want to be a journalist in that country and say anything that's not supportive of the government" [P8]. In such contexts, questions exist as to PV's utility and adaptability to the context and its claim to 'speak truth to power', highlighting the lived experiences of communities and beneficiaries to a wider audience. To address such concerns, practitioners include safeguards: "you need to strike a balance because you don't want to put communities into trouble" [P11].

Communities with local security and conflict issues pose similar challenges. A South American-based practitioner described their experience of working in one such an environment: "if your organization is not well-recognized in these communities, it can be a risk for your staff to carry these kinds of devices" [P10], and the need to "coordinate with key people from the communities, community leaders, to say to the community 'this organization is coming, and collecting this information' in order for the communities to identify us and the environment can be more safe for us" [P10]. Another talked of the challenges of working within refugee contexts, and how "the main challenges would be anonymity of people who are afraid of showing their faces or of being recognized. Because of the sensitivity of the Syria crisis" [P9]. Here PV's claimed qualities of authenticity and directness, and the medium itself (video), can pose a very real risk to the very members of communities it seek to 'empower'. 
Challenges pertaining to the nature of participation of women and girls on PV were described: "in a lot of the countries... [there are regions where] females will never ever participate" [P5] when it comes to using video for data collection, because of cultural views around female representation. This MENA-based practitioner noted that "in the rural regions not many women would like to show their faces" [P9]. Such views were not seen as a fundamental barrier to using PV, but as cultural norms that needed to be navigated, and practitioners developing creative practices to address them. For example, one explained "if a woman has a very interesting story to tell then maybe she can tell it through her voice... people, who don't want to show their faces, we can take the pictures from the back" [P9]. Another described working with a married couople: "she was standing in the back, and she was talking through the mic but we were showing her husband and showing the project that she was talking about" [P9]. While our participants described how the benefits of using PV outweighed the processes required for adaptation, they were aware that "it's not ideal and it's not aesthetically fine" [P9].

\section{Motivation for PV use}

There are varying motives for using PV as a medium among M\&E practitioners. Some use it simply because "some donors require stories" [P4]. However, there is an acknowledgment that the reporting of projects has to cater to multiple stakeholders and multiple hierarchies when working across regions. As such, there are a variety of perceived benefits of using mediums that are accessible to a variety of stakeholders. From the perspective of an M\&E employee based at IFRC headquarters: "As the donor or person sitting back here in [HQ] that's what you want; real life happening and not just the written report" [P8]. For donors, listening to the views of community members is an important as part of the feedback process: "it is more justified to say 'yes, I gave my money to support these people so let us listen to their feedback"' [P4]. Donors and project commissioners even went as far as to suggest this as opening "two-ways communication between us, the project implementer and the community" [P1]. Lest this be seen as a victory for participatory ethos per se, the staff member adds that "I' $m$ not saying a breakthrough or something like that but it will enhance what we already do at the moment" [P1] as the current opportunities for this two-way communication are limited. If the community members wanted to speak with stakeholders such as government officials or donors, they "have to go through several layers to speak with" them. Nevertheless, this is a positive step in building bi-directional communication channels as it serves the agendas of "not only for the organization supporting that change and the back donor but also for the community themselves" [P8] to see changes over time in their situation and how their needs are being met.

\section{An Ongoing Community Voice}

Many participants identified opportunities for ongoing and continuous use of video within their roles: "it could be interesting to capture a flavor of what people felt like at the beginning of the project" [P8]. The use of external evaluators was a less favourable option for many, as the process' outputs "could be shaped by the evaluator's perspective from [HQ]".
They saw the data as being more reliable when the community was fully behind the data collection approach. As the community were playing a key role in PV approaches, it was considered more reliable than "an outside person recording them" [P10]. Giving communities increased agency within data collection strategies was seen as a priority. Some evaluation personnel saw $\mathrm{PV}$ as an approach that enables community members to give them the information they need to receive. In a way this is perhaps with a view to outsourcing data collection to communities, which is also seen as a means of getting more reliable stories: "they are probably going to feel more free to speak and give us more information." [P10]. Time-sensitive assistance was also perceived to be possible with the use of $\mathrm{PV}$, as the communities could potentially learn to use PV and then help Development organizations by sharing timely information, which in turn, could help shape the the assistance provided. This raises a dichotomy with a significant critique of Development work, that the personnel capturing data are detached from the communities they serve: "we just sit in our offices, we plan the things which doesn't even fit them" [P15].

\section{Accountability Towards Communities}

Communities are the arbiters of a successful evaluation. In fact, in many of the contexts our participants work in, communities expect to be partners in the decision making process and "also want to be involved [and] consulted" [P10] rather than being seen as passive recipients of development relief. It is important that the community sees the output of the PV process having a real and practical impact for them, otherwise the efforts put by them into the process would be not as beneficial: "I think the community has to see that the video has taken them to something" [P11]. Participants see the importance of building trust between the community and the organization by delivering outcomes after the video work has gone through the organization's evaluation and decision-making channels. The organization also needs to make sure there is strong communication with partner communities about the remit of their work and how they can help communities achieve their own targets.

\section{Innovating Within Organizational Constraints Organizational Dynamics}

A number of practitioners noted that when building capacity in their organization for new approaches, particularly participatory approaches, "younger... fresh, brand minded, open" [P4] individuals from the organization were prioritized. Picking such motivated people was seen as important for piloting new initiatives as they "would be an amazing champion" for any new initiative [P11]. With motivated personnel on board, the approaches would see greater traction and be passed onto more people. This is an important factor in resource-constrained organizations who often have limited budgets for training and capacity building. Once you have "the right [communication] person who 'gets it", [P12], approaches can be taken up within local chapters with limited resources - where key personnel will maintain momentum within the organization and promote change. The corollary is that there are others who are "resistant to the change" as they are used to "collecting information on paper or in a verbal way", so "you need to talk a lot with them to try to convince of the benefits to have this kind of [approach]" [P10]. Staff are perceived to be 
"struggling with data inputting fatigue" as they are required by numerous internal processes to enter information through different channels, which according to one practitioner ([P5]) results in lack of consolidation and inconsistency of data.

\section{Training, Human Resources and Sustainability}

It has been noted that there is a high turnover of staff within organizations across the Development sector [40], something which our participants confirm as prescient to their context. Frequently, participants mentioned the effects of this in their own work and how they support capacity building across the various tiers in their organization. Some practitioners "involve the regional team in the implementation" [P10] to offset the loss of organizational knowledge which happens when losing team members. Often organizations don't have robust procedures in place to prevent organizational knowledge or capacity depleting due to constant turnover: "we don't have too much time for handovers and some of the things we think are minor but in the end they are really crucial" [P14]. This also applies to the processes that are often not taken into consideration when rolling out new approaches: logistical operations such as translation and organizing training events.

\section{Financial Constraints}

Budgetary considerations we raised as a major factor in deciding to deploy approaches to community feedback within Monitoring and Evaluation. As we have established, there is a considerable appetite for innovation, however personnel are restricted considerably by available resources. Reflecting on video-based approaches, one practitioner commented: "we should not be too naive and make sure we have enough time and we dedicate enough effort to training people " [P5]. Personnel working in M\&E prefer to hire external staff to maintain 'distance" between them and the evaluation. This in effect creates the impression of having an impartial 'auditor' to conduct the role. However, this can be prohibitively expensive and beyond the budget of many local chapters based in resource-constrained environments: " a standard external evaluation costs around 35,000-40,000 USD... we had one evaluation that cost 100,000 USD" [P6], and unsurprisingly organizations struggle to justify such costly approaches. Unfortunately, control is ceded to the budget holder about the type and depth of evaluation work that can take place. One practitioner reflected that depending on "how we sell the tool and how the budget-holders like it" [P14], there would be a possibility of using video evaluation approaches in their existing projects. One participant dismissed many new trends in the humanitarian sector as impractical for many regions: "Using AI \& block-chain in logistics... this is all good but they do not impress those who cannot have this. I find the concept papers very biased towards well-developed NGOs" [P5]. Practitioners do not have the luxury of choosing methods simply on their own merit; they have to think about the applicability of these methods within their unique organizational constraints: " $A l$ ready with the basics people are like 'we don't have the money' or 'we don't have the capacity' so if you say 'oh participatory-' they're like 'woah!' ((laughs))" [P11]. Practitioners described practices that have emerged in response to working in these constraints. One solution could be to use existing tools and platforms that do not require financial outlay on the part of the organization: "they already use WhatsApp [as an] informal way of reporting... would be interesting for us is to formalize and to build on that" [P9]. In a country where there was only one M\&E staff member nationally, regional staff came up with strategies to support them when conducting program evaluations. "We $100 \%$ rely on external support.. you need to fly in people to do the program. There is only one M\&E member!" [P6]. This participant also suggested how to expand the M\&E repertoire of other national chapters, suggesting that within the organization "internal advocacy is still needed!" [P1]. With the help of this internal advocacy, current donors and other stakeholders can be shown the merits of new approaches and the need to allocate resources to them: "our role is to guide the rest of the organization.. showcase different uses of video" [P11]. While limited by currently available approaches, they nevertheless express an appetite innovative forms of community engagement and a desire to set an example for the rest of the organization.

\section{A VISION OF PV 2.0}

Through reflection on the aforementioned themes around PV use in NGOs in light of current industry practice, we can present the new vision of PV 2.0. Object Based Media (OBM) is a key industry practice, emerging in response to the complexities of the new media landscape. OBM can help frame the design of production tools, processes and technologies to support media production within this space. In OBM, each piece of video (and audio) is accompanied by rich metadata which describes the content [29], together forming an 'object'. This metadata could be anything from the technical specification of the camera, to semantic descriptions of what occurred in the video. By producing, editing, consuming and distributing these media 'objects', it is possible to support new forms of production and consumption scenarios, in which objects are assembled into narratives depending on a variety of factors [51]. Key properties of OBM can inform our vision of PV:

Removing Workflow Assumptions: OBM removes many of the assumptions of traditional linear production workflows which rely on the uni-directional transfer of video content from one process to the next. In these new processes, production roles are flexible and interchangeable, where consumers can be producers and editorial decisions can be made at any point in the process.

Metadata is King: OBM considers all content equal, through the application of rich metadata. It is by querying and manipulating these data that new content is shaped and distributed, in contrast to the traditional model of 'cutting' up videos in a destructive editing process which produces a single output.

Reconfigurability that Supports New Production Scenarios: OBM natively supports emergent forms of consumption such as interactive narratives [21], branching narratives (e.g. choose-your-own-adventure) [2] and second-screen content (e.g. TV show apps) [28]. Individual elements of video can be repackaged in myriad forms based on any number of factors e.g. personal preference, contextual relevance, or language.

By developing new practices and processes based on the potential of $\mathrm{OBM}$ and the increasingly complex requirements 
of commissioning organizations, we believe PV 2.0 can become a transformative method of community engagement and put forward recommendations on how this can be achieved. These recommendations are informed by our reflections on the findings, through the lens of OBM.

\section{Redefining Roles and Process}

\section{Redefining Production Roles}

As our NGO participants attest, the financial investment in broadcast equipment for PV using current practices is substantial. Media production technology has advanced considerably since the camcorders and editing suites leveraged by the Fogo process, or popularized by Lunch and Lunch in the late 90s [30]. Contemporary approaches have promoted the use of consumer devices and software [3] which allows for lower cost of deployment. However despite using everyday technologies, our participants' approach was still underpinned by the preconceived constraints of linear production pipeline they worked within (one could argue that even invoking the metaphor of pipelines suggests a linear framing transfixed on uni-directional flows of information).

The traditional PV pipeline assumes roles such as 'presenters', 'producers', 'editors' and 'consumers'. Yet, there could be tremendous value in the community video participation space where these distinctions are removed. This is particularly true in the context of reflective or longitudinal use of $\mathrm{PV}$, where facilitators can work on increasing media literacies required for each of these roles as part of the group-work that is conducted. As these production roles blur, the age of the prosumer is dawning upon those who are working in NGO field contexts. The thrust of this argument has emerged not from visionary ideals of technology creators (whether based in the West or otherwise), but rather media praxis of populations in Developing contexts, a glimpse of which was shown earlier in this paper. We argue that there are ingrained assumptions within PV practice about which tasks within the PV production pipeline can be done by 'novice' communities [10] and which tasks are within reach. In PV practice, communities are encouraged to engage in the story ideation and video capture phases of the pipeline. Some current approaches give selected community members additional training or a chance to take part in the editorial and curator-ship stages. Fewer approaches still involve community members in post-production processes (i.e. adding music and titles). Furthermore, these later stages are almost always under the tutelage of 'professionals'.

There is a clear need for NGOs to meet the challenges of complex stakeholder requirements of content. Yet, a cautionary approach is taken towards innovation of PV methods, as practitioners have made an investment into their current community video work approaches. We stress that this is not due to an inherent desire to suppress community participation. Rather these are pragmatic arrangements in light of perceived constraints in communities' technical skill and media literacy, that are required to perform some of these steps. However, while this assumption was undoubtedly true in the days of non-digital production, in the age of ubiquitous consumer technologies, this assumption is less valid. With the marked increase in media (and technology) literacy, participants in PV processes can spend more time focusing on the act of telling their stories effectively and thus produce richer stories, rather than being bogged down in complex, technical tasks. However, challenges do remain in how NGOs can facilitate such tasks among populations with varying media literacies.

In PV2.0, activities traditionally seen as single-person tasks (such as editing on a linear timeline) can become social, group based activities in which the editing system acts on behalf of the group. Through the use of non-destructive objects, multiple editorial viewpoints can be represented, and participants can take ownership over different parts of an edit. Such opportunities may lie in utilizing companion screens to perform time consuming tasks in parallel [8], or leveraging multiple devices to allow a richer experience of video consumption [45]. By removing assumptions placed on the workflow by antiquated technology limitations, we give agency back to the practitioners and communities that they work with. This allows them to form their own practices and processes to best meet their requirements, developed through iterative approaches [12] not limited to video. OBM's foundational emphasis on generating rich content metadata supports the recording of provenance, contribution and consent. These are key to making use of content in PV2.0, enabling organizations to commission data that are more authentic representations of communities.

\section{Redefining the Editorial Process}

Redefining the PV process in terms of re-configurable objects forces further reflection on the role of the editorial and post-production processes normally associated with PV. A particular tension is the presentation of a 'final edit', an artifact which is seen as the end result of the traditional media production pipeline. Current PV methodologies engage participants in performing the roles required to generate this artifact. There is a clear, established, and shared understanding of who has editorial control of this final edit's contents (deciding what content is/isn't relevant to the narrative). The content in such outputs (e.g. clips and audio) can and does get taken out of context, reconfigured after-the-fact by persons who may chose to disregard the original narrative and editorial decisions undertaken within the well-defined PV workflows used by practitioners.

Organizations are aware of this significant issue, and the perceived risks associated with such actions: brand reputational damage and breakdown in community trust due to misinterpretation of community voice. Worse still, in sensitive contexts, media taken out of context can inflame tensions between community groups and/or political actors. Current PV practice dictates that facilitators ensure the community validates and approves the final edit before leaving the community context. However, by introducing new processes that produce inherently re-configurable content, we need to promote and maintain the authenticity of community voice within the media outputs of NGO field processes, a driving principle behind the rise of PV. There are a number of ways in which these acts of maintaining and promoting authenticity manifest themselves in practice. There is an implicit trust that underlies the handling of the PV output once the NGO or facilitators leave the field. That is, they are committed to represent the community 
as the community themselves wanted to be represented. In other words, the community expects the media content to not be used maliciously or to misinterpret community views and practices. However, presently, the community have no mechanism for reporting or invalidating their consent over the use of the media once it leaves the field context.

Editorial content is thus concentrated into the hands of professionals out of a desire to safeguard both the community and the message, particularly in sensitive contexts where organizations are keenly aware of the do no harm principle $[37,1]$. Therefore, to rethink PV in light of contemporary media practices is not to ignore the legitimate concerns about trusting sources, authentic representation and participant consent, but rather to bring these concerns into dialogue with the way the media landscape is changing. In fields such as professional journalism, where trust is based on agreed upon editorial standards and reporting mechanisms, flouting these principles results in serious ramifications for the publisher and journalist. However, where agency is returned to the community in defining and capturing their own narrative (in the context of media objects that can be reconfigured after-the-fact), we need to be clear about how to maintain notions of trusted sources, editorial standards and reporting mechanisms that can underpin practice. A number of prominent PV practitioners and researchers have recommended frameworks for such practice and statements of ethics $[6,32,44,30]$. These ethical guidelines, which acknowledge the danger of the mediums that they work with, nevertheless exhort the benefits of using them in managed, controlled ways. Seeking to rein in the infinite possibilities of un-constrained re-use of media objects, narrative [50], semantic [38] or consent constraints [36] could be encoded by the community during the PV process. A simplistic example of this might be displaying metadata on each object so that provenance and context can be scrutinized [13]. However we recognize the nuanced requirement to balance audience direction and the narrative intent of PV facilitators, NGOs, communities [5] and individual participants.

In PV2.0 where content can be reconfigured and consumed in un-imagined ways after leaving the community, we need mechanisms to maintain trust and editorial control. These processes need to acknowledge that communities do not provide 'blanket' consent to how their content is used, and that they have the right to re-vet content presented in new ways. These new mechanisms should be based on the native provenance information within the metadata produced in an OBM enabled workflow [36].

\section{The Potential of PV 2.0}

\section{New Consumption Models}

As reported by our participants, commissioners of content are increasingly under pressure to produce outputs which meet the requirements of multiple stakeholders. However the linear production models promoted by current PV practice are not suited to delivering multiple narratives in multiple formats to multiple stakeholders. Under OBM, video can be compared, contrasted and illustrated with or by other data sets. Interactive documentaries (iDocs) [22] offer an obvious example of how to open up spaces for rich interaction with and interpretation of content [18], external data sources [12] and the potential to represent multiple authorial voices via different modes of participation [19]. The production of an iDoc is predicated on rich OBM being produced by the community, and represents an evolving document that supports longitudinal deployment of PV in a community. As new data is produced or sourced by the community or organization, this data is curated and included into the iDoc, updating or augmenting narratives that are available to audiences. Practical challenges remain however e.g. where data is stored, and who is responsible for its maintenance; issues which need to be explored within PV2.0 workflows.

In PV2.0 the reliance on a linear production pipeline is replaced with a flexible interpretation of narrative consumption. This allows communities to produce content that can be consumed by multiple stakeholders (e.g. through an iDoc format), whilst maintaining narrative and editorial control over the content. Mechanisms for facilitating the co-production and semantic narrative of such formats however do not yet exist. Such processes will need to support co-production and continual feedback loops between facilitators, community and audience, but will allow for rich dialog between the community and external audiences.

\section{Maintaining Authenticity at Scale}

As the opportunity for producing and distributing higher volumes of media content from communities all over the world becomes a reality, the challenges of translation and interpretation become significant. Aside from the reality: that working directly with communities in field contexts necessitates the capture of multiple languages and dialects; the fact that this content is not curated down into a single output (as in current PV practice), means that much more content potentially needs to be translated or contextualized. The reconfigurability and flexibility of the OBM model operates under the assumption that objects can be understood in their entirety at any point in the future: to support assemblage of new consumption experiences. However, it is impractical to assume such granularity of translation (or transcription) from field PV deployments. Thinking in terms of OBM, however, does allow us to consider how to support practical engagement with the process of translation. Under the assumption that no content is ever destroyed, original audio tracks can be maintained with content, with translations being added as additional (or optional) synced content. This allows for both accurate future translation, and also for validation of the translation at any point in the future. We do acknowledge however, that challenges still remain around issues of the ethics of language translation and the possible introduction of bias when multiple stakeholders are involved in this process.

In PV2.0, production processes need to be designed with the assumption of future re-use of content. Language and translation are core issues for re-use which need addressing. Advances in machine learning and automatic translation offer potential to bootstrap these processes, however fundamentally, media objects need to retain the original content to facilitate transparency and authenticity in later reconfiguration. 


\section{PV Video as Data}

There are core epistemological questions being asked about the nature of the data that PV produces. Present day commissioners of PV are recognizing the rich value of video as data, and expecting to utilize those videos in ways never envisioned by original proponents of PV. As our findings demonstrate, the resource intensive nature of PV production methods force organizations into using the output for many different purposes and audiences (back-donors vs. headquarters). However, these audiences have different expectations and requirements of the content. PV is being actively used and promoted by these organizations as 'data sources' for reporting, however our participants intimated that due to lack of established protocols around the analysis of PV data, approaches that are currently used lack sufficient analytical rigor. While organizations are keen to embrace labels such as data driven, the practical constraints faced mean that program coordinators feel unsupported in utilizing video data for advocacy or project reporting. This is perhaps unsurprising as video represents a small proportion of the data that NGOs generate and process.

Strategies to conduct media analysis include using crowdsourced annotation, such as used by previous researchers in NGO field contexts $[20,23,7,41]$ and working with panels of visual analysis experts, leveraging their skills to train and equip PV practitioners. Crowdsourcing suggestions, nevertheless, are not sustainable in the long term. Under this new assumption that the output of the process (video) will be interpreted natively as data, PV2.0 could configure production processes to ask different questions of the community in order to create video that is more suitable for use as part of this triangulation. This could be augmented by engaging the community in performing elements of the analysis - by breaking down the skill barriers required in this practice, for example, by enabling them to tag content during the production processes.

Being data driven has become synonymous with the increasing use of quantitative data in current reporting practice (no doubt driven by a desire to validate and justify their approaches). Unfortunately, as reported by our participants, this results in video data also being incorrectly turned into quantitative data e.g. counting number of people that appeared in a video as a measure of project participation. While the visual arts and media studies disciplines have studied the inherent value of video in depth, practitioners we encountered seemed to not be well-versed in such approaches [25, 26]. Instead of considering how to convert video into data (quantitative or otherwise) and risk losing the intrinsic values that video as a modality offers analysts, in PV2.0 we see triangulation of video with other available data (both primary and secondary data) sources being key to building understanding. This, however, requires additional contextual information to be included consistently as part of the media object creation process, which requires increased willingness and rigor on the part of media creators (or commissioners).

Current PV practice places less emphasis on the process of production as a valuable data source. In reality, artefacts generated throughout the process (e.g. workshop artifacts (storyboards), group discussions, and unused edits) are valid data sources present within the various stages of the PV process that are discarded in favor of the 'final edit'. These data are discarded partly due to a lack of procedures for capturing this information, but predominantly because their value for the organization is not realized. Furthermore, when considering how multiple narratives can be captured by the community in PV 2.0, there are vital data that exists in the process of how and what criteria the community used to arrive at particular curatorship decisions. PV2.0 should support practitioners in capturing these decision-making process outputs as part of its workflows from the start, rather than treating these as secondary (and invalid) data. These decisions within the creative process, made by participants, are key to understanding and contextualizing the final output. Key examples include: decisions on what they chose to include in the narrative (and explicitly stating what was excluded), who made these decisions (made by community representative or group decision), and the process by which they came to make them. These are valuable data points that could better contextualize the video's message. Nevertheless, this requires a commitment to PV2.0's methodology on the part of the facilitator, and less rigorous applications of the process would still result in 'less useful' data.

PV2.0 configures the production process to ask different questions of the community in order to create video data that is more suitable for use as part of data triangulation. Key to enabling this process is both capturing the process as data, and engaging the community in performing analysis of content - by breaking down the skill barriers required in this practice: for example, by enabling them to tag content during the production process.

\section{CONCLUSION}

We engaged practitioners from a large networked NGO in conversations around the potentials, challenges and affordances of current Participatory Video (PV) practice in their work as International Development professionals within community feedback and M\&E. Our findings suggested a number of barriers to applying current PV practice including social, economic and political realities that shaped the way PV is perceived and used in NGO contexts. We found an incongruity between emergent practices in the contemporary media landscape and media as currently utilized within NGO contexts. In response to these realities, we propose a vision for PV 2.0, discussing the opportunities and challenges that emerge when reconsidering PV in light of emergent industry practice e.g. increased metadata use. We posit that the existing presumptions of low media and technology literacies hold little sway when considering how communities can be empowered to create more authentic and meaningful content.

\section{ACKNOWLEDGMENTS}

This research was funded by the EPSRC Digital Economy Research Center (EP/M023001/1) and the EPSRC CDT in Digital Civics (EP/L016176/1). Data from this publication is openly available under an 'Open Data Commons Open Database License'. Additional metadata are available at: http: //dx.doi.org/10.25405/data.nc1.c.4808403. 


\section{REFERENCES}

[1] Mary B Anderson. 1999. Do no harm: how aid can support peace or war. Lynne Rienner Publishers, Boulder, Colorado.

[2] Tom Bartindale, Guy Schofield, and Peter Wright. 2016. In Proceedings of the 2016 CHI Conference on Human Factors in Computing Systems - CHI '16. ACM. DOI : http://dx.doi.org/10.1145/2858036.2858102

[3] Tom Bartindale, Delvin Varghese, Guy Schofield, and Miki Tsukamoto. 2019. Our Story: Addressing Challenges in Development Contexts for Sustainable Participatory Video. In Proceedings of the 2019 CHI Conference on Human Factors in Computing Systems (CHI '19). Association for Computing Machinery, New York, NY, USA, Article Paper 437, 12 pages. DOI : http://dx.doi.org/10.1145/3290605.3300667

[4] Frank Bentley and Danielle Lottridge. 2019. Understanding mass-market mobile TV behaviors in the streaming era. Conference on Human Factors in Computing Systems - Proceedings 1 (2019), 1-11. DOI: http://dx.doi.org/10.1145/3290605.3300491

[5] Pablo Cesar and Konstantinos Chorianopoulos. 2008. Interactivity and user participation in the television lifecycle. (2008), 125. DOI :

http://dx.doi.org/10.1145/1453805.1453830

[6] Vivian Chávez, Barbara Israel, Alex J Allen III, Maggie Floyd DeCarlo, Richard Lichtenstein, Amy Schulz, Irene S Bayer, and Robert McGranaghan. 2004. A bridge between communities: Video-making using principles of community-based participatory research. Health Promotion Practice 5, 4 (2004), 395-403. DOI : http://dx.doi.org/10.1177/1524839903258067

[7] Kuang Chen, Akshay Kannan, Yoriyasu Yano, Joseph M Hellerstein, and Tapan S Parikh. 2012. Shreddr: pipelined paper digitization for low-resource organizations. In Proceedings of the 2nd ACM Symposium on Computing for Development. ACM, 3. DOI : http://dx.doi.org/10.1145/2160601.2160605

[8] Andy Cockburn and Tony Dale. 1997. CEVA: a tool for collaborative video analysis. GROUP 97 (1997), 47-55. DOI : http://dx.doi.org/10.1145/266838.266857

[9] Sebastien Cuendet, Indrani Medhi, Kalika Bali, and Edward Cutrell. 2013. VideoKheti. In Proceedings of the SIGCHI Conference on Human Factors in Computing Systems - CHI'13. ACM Press, 2833. DOI : http://dx.doi.org/10.1145/2470654.2481392

[10] Nicola Dell and Neha Kumar. 2016. The Ins and Outs of HCI for Development. Proceedings of the 2016 CHI Conference on Human Factors in Computing Systems (2016), 2220-2232. DOI :

http://dx.doi.org/10.1145/2858036.2858081

[11] Nicola Dell, Vidya Vaidyanathan, Indrani Medhi, Edward Cutrell, and William Thies. 2012. Yours is better!: participant response bias in HCI. In Proceedings of the SIGCHI Conference on Human Factors in Computing Systems. ACM, 1321-1330. DOI: http://dx.doi.org/10.1145/2207676.2208589

[12] Mirka Duijn and Hartmut Koenitz. 2017. Beyond the timeline a data-driven interface for interactive documentary. TVX 2017 - Adjunct Publication of the 2017 ACM International Conference on Interactive Experiences for TV and Online Video (2017), 33-37. DOI : http://dx.doi.org/10.1145/3084289.3089920

[13] fourcornersproject.org. 2018. Four Corners Project. https://fourcornersproject.org/en/about/. (2018). [Online; accessed 19-July-2019].

[14] David Frohlich, Simon Robinson, Kristen Eglinton, Matt Jones, and Elina Vartiainen. 2012. Creative cameraphone use in rural developing regions. Proceedings of the 14th international conference on Human-computer interaction with mobile devices and services - MobileHCI '12 September 21-24 (2012), 181. DOI : http://dx.doi.org/10.1145/2371574.2371603

[15] David M. Frohlich, Dorothy Rachovides, Kiriaki Riga, Ramnath Bhat, Maxine Frank, Eran Edirisinghe, Dhammike Wickramanayaka, Matt Jones, and Will Harwood. 2009. StoryBank. In Proceedings of the 27th international conference on Human factors in computing systems - CHI 09. ACM Press, New York, New York, USA, 1761. DOI : http://dx.doi.org/10.1145/1518701.1518972

[16] globalwebindex.com. 2017. GlobalWebIndex - Digital Analytics, Audience Insight Tools and Trends. https://www.globalwebindex.com/. (2017). [Online; accessed 19-July-2019].

[17] grabyo.com. 2019. Global Video Trends Report 2019. https://about.grabyo.com/wp-content/uploads/2019/07/ Grabyo-Global-Video-Trends-Report-2019.pdf. (2019). [Online; accessed 19-July-2019].

[18] David Philip Green, Clara Crivellaro, and Jimmy Tidey. 2015. Interactive Design Documentary as a Method for Civic Engagement. In TVX 2015. 161-166. DOI : http://dx.doi.org/10.1145/2745197.2755518

[19] David Philip Green, Guy Schofield, Gary Pritchard, Patrick Olivier, and Peter Wright. 2017. Cinehacking Cape Town - Embracing Informality in Pursuit of High Quality Media. Proceedings of the 2017 CHI Conference on Human Factors in Computing Systems CHI' 172010 (2017), 4753-4764. DOI : http://dx.doi.org/10.1145/3025453.3025481

[20] Aakar Gupta, William Thies, Edward Cutrell, and Ravin Balakrishnan. 2012. mClerk: enabling mobile crowdsourcing in developing regions. In Proceedings of the SIGCHI Conference on Human Factors in Computing Systems. ACM, 1843-1852. DOI : http://dx.doi.org/10.1145/2207676.2208320 
[21] Jonathan Hook. 2018. Facts, interactivity and videotape: Exploring the design space of data in interactive video storytelling. TVX 2018 - Proceedings of the 2018 ACM International Conference on Interactive Experiences for TV and Online Video (2018), 43-55. DOI :

http://dx.doi.org/10.1145/3210825.3210826

[22] i-docs.org. 2017. About i docs. http://i-docs.org/about-idocs. (2017). [Online; accessed 19-July-2019].

[23] Esther Jang, Mary Claire Barela, Matt Johnson, Philip Martinez, Cedric Festin, Margaret Lynn, Josephine Dionisio, and Kurtis Heimerl. 2018. Crowdsourcing Rural Network Maintenance and Repair via Network Messaging. In Proceedings of the 2018 CHI Conference on Human Factors in Computing Systems. ACM, 67. DOI: http://dx.doi.org/10.1145/3173574.3173641

[24] Jarvey, Natalie. 2017. Mark Zuckerberg Details Facebook's "Video First" Strategy. https://www.hollywoodreporter.com/news/facebookshares-up-revenue-growth-970957. (2017). [Online; accessed 19-July-2019].

[25] Hubert Knoblauch, Alejandro Baer, Eric Laurier, Sabine Petschke, and Bernt Schnettler. 2008. Visual analysis. New developments in the interpretative analysis of video and photography. In Forum Qualitative Sozialforschung/Forum: Qualitative Social Research, Vol. 9.

[26] Hubert Knoblauch and Bernt Schnettler. 2012. Videography: Analysing video data as a 'focused'ethnographic and hermeneutical exercise. Qualitative Research 12, 3 (2012), 334-356. DOI : http://dx.doi.org/10.1177/1468794111436147

[27] Neha Kumar, Pallavi Lal, Richard Anderson, Trevor Perrier, Michelle Desmond, Kiersten Israel-Ballard, Vikrant Kumar, Sudip Mahapatra, Anil Mishra, Shreya Agarwal, and Rikin Gandhi. 2015. Projecting health. In Proceedings of the Seventh International Conference on Information and Communication Technologies and Development - ICTD '15. ACM Press, New York, New York, USA, 1-10. DOI:

http://dx.doi.org/10.1145/2737856.2738023

[28] Jie Li, Thomas Röggla, Maxine Glancy, Jack Jansen, and Pablo Cesar. 2018a. A new production platform for authoring object-based multiscreen TV viewing experiences. TVX 2018 - Proceedings of the 2018 ACM International Conference on Interactive Experiences for TV and Online Video (2018), 115-126. DOI :

http://dx.doi.org/10.1145/3210825.3210834

[29] Jie Li, Zhiyuan Zheng, Britta Meixner, Maxine Glancy, Pablo Cesar, and Thomas Röggla. 2018b. Designing an object-based preproduction tool for multiscreen TV viewing. Conference on Human Factors in Computing Systems - Proceedings 2018-April (2018), 1-6. DOI: http://dx.doi.org/10.1145/3170427.3188658
[30] Chris Lunch and NIck Lunch. 2006. Insights into Participatory Video. Technical Report. InsightShare. 126 pages. DOI:

http://dx.doi.org/10.1111/j.1467-7660.2008.00473_13.x

[31] Jennifer Manuel, Geoff Vigar, Tom Bartindale, and Rob Comber. 2017. Participatory Media: Creating Spaces for Storytelling in Neighbourhood Planning. In Proceedings of the 2017 CHI Conference on Human Factors in Computing Systems - CHI '17. ACM Press, New York, New York, USA, 1688-1701. DOI: http://dx.doi.org/10.1145/3025453.3025745

[32] Elizabeth-Jane Milne, Claudia Mitchell, and Naydene De Lange. 2012. Handbook of participatory video. AltaMira Press, Lanham, Md.

[33] ooyala.com. 2018. Global Video Index Q2 2018. http://go.ooyala.com/rs/447-EQK-225/images/OoyalaGlobal-Video-Index-Q2-2018.pdf. (2018). [Online; accessed 19-July-2019].

[34] O'Dubhghaill, Liam and O'Gorman, Mary and Flanagan, Patrick. 2013. The Prosumer I Role of the author/ audience within digital new media. https://authorandaudience.wordpress.com/the-prosumer/. (2013). [Online; accessed 19-July-2019].

[35] participatoryvideo.com. 2013. PV History: Colin Low The Children Of Fogo Island. http://www.participatoryvideo.com/2013/02/pv-historycolin-low-the-children-of-fogo-island/. (2013). [Online; accessed 09-September-2019].

[36] Perez, Sarah. 2017. Spotify acquires blockchain startup mediachain to solve music's attribution problem. https://techcrunch. com/2017/04/26/spotifyacquiresblockchain-startup-mediachain-to-solve-musicsattribution-problem. (2017). [Online; accessed 09-August-2019].

[37] James Putzel. 2010. Do no harm: international support for statebuilding. Organisation for Economic Co-opertation and Development, Paris, France.

[38] Elena Rishes, Stephanie M. Lukin, David K. Elson, and Marilyn A. Walker. 2013. Generating Different Story Tellings from Semantic Representations of Narrative. In Interactive Storytelling. Springer International Publishing, Cham, 192-204. DOI :

http://dx.doi.org/10.1007/978-3-319-02756-2_24

[39] Tony Roberts. 2016. Women's Use of Participatory Video Technology to Tackle Gender Inequality in Zambia's ICT Sector. Proceedings of the Eighth International Conference on Information and Communication Technologies and Development - ICTD '16 (2016), 1-11. DOI :

http://dx.doi.org/10.1145/2909609.2909673

[40] Laura Roper and Jethro Pettit. 2002. Development and the learning organisation: an introduction. Development in Practice 12, 3-4 (2002), 258-271. DOI : http://dx.doi.org/10.1080/0961450220149654 
[41] Navkar Samdaria, Ajith Sowndararajan, Ramadevi Vennelakanti, and Sriganesh Madhvanath. 2013. Cyber cafes as microtasking hubs. In Proceedings of the 11th Asia Pacific Conference on Computer Human Interaction. ACM, 364-370. DOI : http://dx.doi.org/10.1145/2525194.2525305

[42] Seale, Shelley. 2018. Mobile Video Consumption Tech Trends for 2019. https://www.stateofdigitalpublishing.com/insights/ mobile-video-consumption/. (2018). [Online; accessed 19-July-2019].

[43] Jacqueline Shaw. 2016. Emergent ethics in participatory video: negotiating the inherent tensions as group processes evolve. Area 48, 4 (2016), 419-426. DOI : http://dx.doi.org/10.1111/area.12167

[44] Jackie Shaw and Clive Robertson. 2008. Participatory video: A practical approach to using video creatively in group development work. Routledge. DOI : http://dx.doi.org/10.4324/9780203133217

[45] Davy Smith, Tom Bartindale, Jonathan Hook, Gerard Wilkinson, Guy Schofield, Phil Stenton, Marian F. Ursu, and Matthew Brooks. 2017. Designing reconfigurable televisual experiences. DIS 2017 Companion Proceedings of the 2017 ACM Conference on Designing Interactive Systems (2017), 368-371. DOI: http://dx.doi.org/10.1145/3064857.3064867

[46] David R Thomas. 2006. A general inductive approach for analyzing qualitative evaluation data. American journal of evaluation 27, 2 (2006), 237-246. DOI : http://dx.doi.org/10.1177/1098214005283748

[47] Timson, Evelyn. 2018. Strategy: Digital Video Content Trends. https://www.mel twater.com/blog/digital-video- content-trends-in-2018/. (2018). [Online; accessed 19-July-2019].

[48] Crystal Tremblay and Ana Maria Peredo. 2014. Participatory video as an approach for strengthening collective social entrepreneurship: The recycling cooperative movement in brazil. Research Methodology in Strategy and Management 9, August (2014), 189-214. DOI :

http://dx.doi.org/10.1108/S1479-838720140000009015

[49] UNDP. 2009. Handbook on Planning, Monitoring and Evaluating. Undp (2009), 1-232.

[50] Gerard Wilkinson, Tom Bartindale, Tom Nappey, Michael Evans, Peter Wright, and Patrick Olivier. 2018. Media of Things: Supporting the Production of Metadata Rich Media Through IoT Sensing. In Proceedings of the 2018 CHI Conference on Human Factors in Computing Systems (CHI'18). ACM, New York, NY, USA, Article 206, 13 pages. DOI : http://dx.doi.org/10.1145/3173574.3173780

[51] Williams, Doug and Wyver, John and Glancy, Maxine. 2016. Evaluating the potential benefits of object $\neg$-based broadcasting. Technical Report. British Telecom, London, UK. https://nem-initiative.org/wp-content/ uploads/2016/11/doug-willams-obb.pdf

[52] Kehkashan Zeb, Stephen Lindsay, Suleman Shahid, Waleed Riaz, and Matt Jones. 2019. Sugar Ka Saathi-A Case Study Designing Digital Self-management Tools for People Living with Diabetes in Pakistan. In IFIP Conference on Human-Computer Interaction. Springer, 161-181. DOI :

http://dx.doi.org/10.1007/978-3-030-29387-1_10 